\title{
Selective Automation Upgrade in Distribution Networks Towards a Smarter Grid
}

\author{
Aggelos S. Bouhouras, Georgios T. Andreou, Member, IEEE, Dimitris P. Labridis, Senior Member, IEEE, and \\ Anastasios G. Bakirtzis, Senior Member, IEEE
}

\begin{abstract}
Research on smart grid technologies has been advancing over the last years, producing novel practices concerning mainly the power distribution networks. However, in many countries these networks still operate in their traditional form, without offering the real-time operational characteristics which are essential for the utilization of the aforementioned practices. On the other hand, due to the extent of urban power distribution networks, as well as the substantial cost of medium voltage equipment, the full upgrade of these networks is in most cases not a feasible option. In this work, alternative options of selective automation upgrade in power distribution networks are offered, corresponding to the desired operational status of these networks. More specifically, the essential upgrades are analyzed for the implementation of reliability improvement and loss reduction techniques on such a network.
\end{abstract}

Index Terms-Power distribution, loss reduction, reliability improvement, network upgrade.

\section{INTRODUCTION}

$\mathbf{T}$ HE CONCEPT OF smart grids has received a lot of attention over the last few years. Substantial research has already been conducted on it, and even more is yet to come. Novel technologies are developed, and novel services are envisioned, aiming to provide power systems in general and power distribution networks (DNs) in particular with an unprecedented level of automation and intelligence.

The key of smart grids, the prerequisite for their implementation, is information. In order to program and utilize automated actions on the power grid, the continuous knowledge of its operational characteristics throughout its whole extent is essential. Apart from that, the concept of utilizing automated actions on the grid also presupposes the existence of automated switchgear at its nodes, which will moreover enable the remote handling of the system by its administrators.

These two prerequisites comprise at the same time the greatest barrier that prohibits the large scale implementation of the smart grid paradigm. This problem aggravates even more in the case of medium voltage (MV) DNs, mainly due to their extent and substantial branching. Because of these factors, for any given network there are a relatively large number of nodes

Manuscript received September 10, 2010; accepted September 14, 2010. Date of publication October 21, 2010; date of current version November 19, 2010. Paper no. TSG-00126-2010.

The authors are with the Department of Electrical and Computer Engineering, Aristotle University of Thessaloniki, Thessaloniki 541 24, Greece (e-mail: bakiana@eng.auth.gr).

Color versions of one or more of the figures in this paper are available online at http://ieeexplore.ieee.org.

Digital Object Identifier 10.1109/TSG.2010.2080294 that have to be monitored, in order to continuously obtain the network's operational characteristics. Combined with the increased cost of metering equipment for the MV level, due to the measurement transformers involved, this issue makes the cost prohibitive for the deployment of the essential equipment in order to fully monitor such a network [1].

The scope of this paper is to provide a framework for the determination of techno-economically viable selective upgrades in power distribution networks, according to prespecified target actions. More specifically, in Section II the traditional structure of contemporary MV DNs is analyzed, and a typical network segment is used in order to provide an indication concerning the cost involved for its full automation upgrade. Subsequently, in Section III procedures are provided for the determination of the essential selective automation upgrade in MV DNs, with respect to reliability improvement and loss reduction. In Section IV, these procedures are presented as alternative upgrade options for a real MV DN segment, in examples aiming to offer an indication of the different operational benefits that may result according to different selective upgrades. Finally, in Section V the conclusions of the work are presented.

\section{AUtOMATION Upgrade IN MV POWER DISTRIBUTION NETWORKS}

\section{A. MV Power Distribution Networks}

Urban MV DNs in Greece consist of groups of interconnected radial circuits, as shown in Fig. 1. The urban areas are supplied with power through high voltage (HV) power lines, which transmit the power from power plants. These power lines end typically in HV/MV power substations, which in turn feed the urban power distribution networks. The power lines interconnecting these substations may be underground cables or overhead lines.

Considering a single feeder, as it can be observed in Fig. 2 it will consist of a series of step-down transformers $(20 / 0.4 \mathrm{kV})$, interconnected in most cases of urban DNs by underground cables. The feeder is connected at both ends to a HV/MV power substation, so as to be able to be supplied with power from two alternative points. In both ends there is a MV circuit breaker, one normally closed (NC) during operation and one respectively normally open (NO). The MV/LV transformers are equipped with manual load switches.

In such feeders, measuring capabilities exist only at the level of the HV/MV power substations located at the ends of the feeder. The respective distribution system operator (DSO) has to estimate the condition of the whole network based on these 


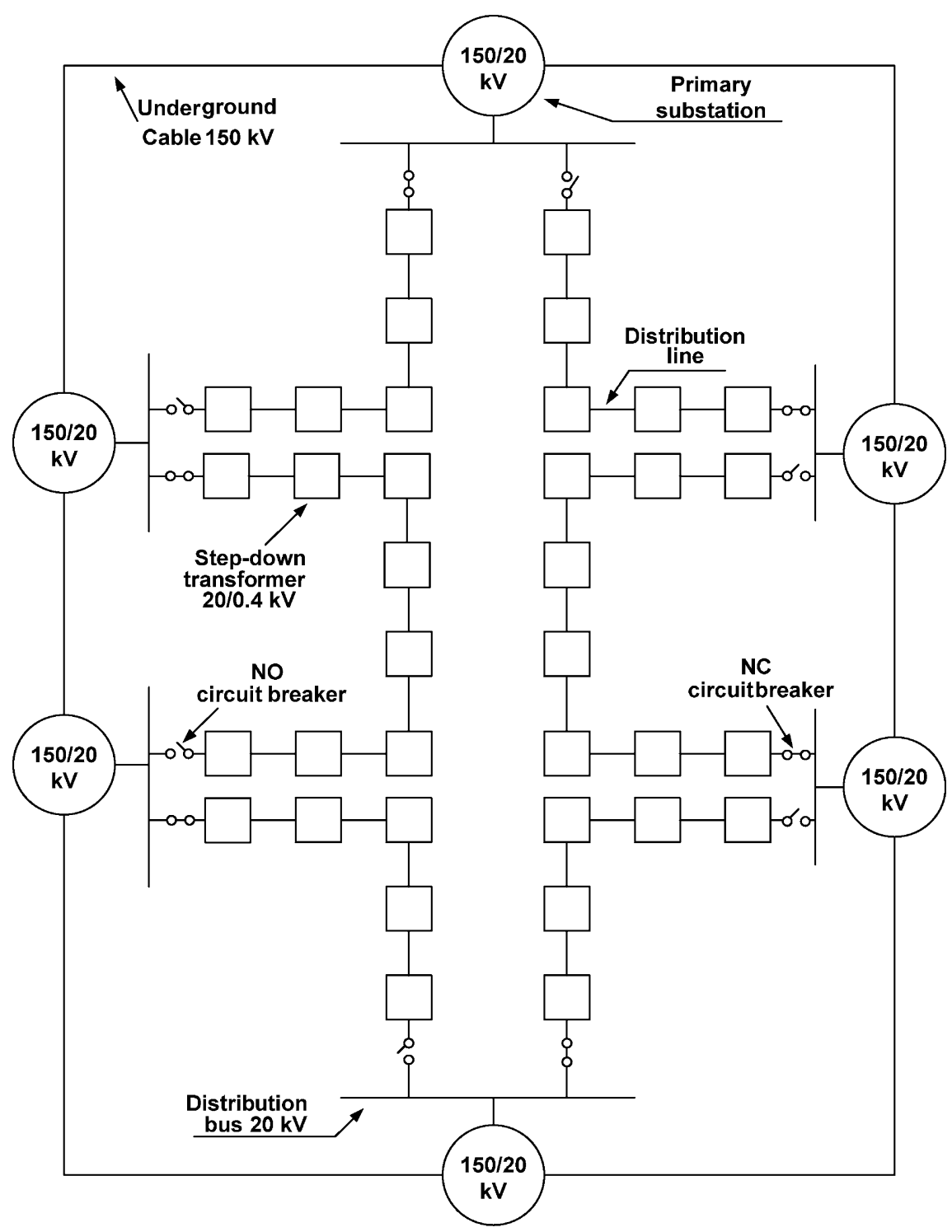

Fig. 1. Typical topology of MV power distribution networks in Greece.

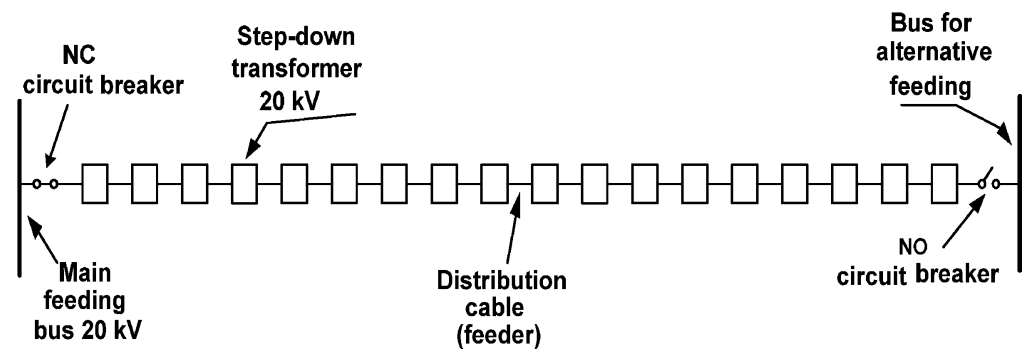

Fig. 2. Typical layout of an urban distribution feeder.

measurements. This task is of course rather complicated, considering the fact that urban DNs extend to hundreds of kilometers of power lines, due to the large number and density of the urban power consumptions.

DSOs have inevitably deployed a number of practical solutions in order to overcome the difficulties resulting from the lack of measurements. Public Power Corporation (PPC) for example, the company who has acted until now as local DSO in Greece, estimates the load of each MV/LV step-down transformer by dividing the total load of the respective feeders among all its step-down transformers in proportion to their rated power. However, this approximation may be valid for the planning of network expansion, but it is far from reliable when it comes to real-time operations.

\section{B. Automation Upgrade}

In order to obtain real-time information concerning the operation of an urban DN, the deployment of specific equipment 


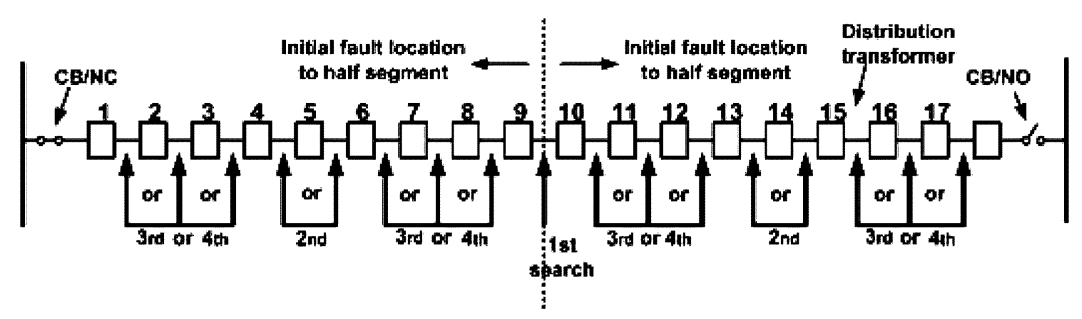

Fig. 3. Possible manual interferences for fault isolation.

at all its nodes is essential. This equipment includes fundamentally for each node of the network (i.e., each MV/LV step-down transformer) [1]:

- Three current transformers for the measurement of all phase currents (typical cost: $2550 €$ )

- Three voltage transformers for the measurement of all phase voltages (typical cost: $3750 €$ )

- One intelligent electronic device (IED) for the actual measurements (typical cost: $1000 €$ )

Apart from that, a communication system will have to be deployed at each transformer, so as to transmit the measurements to the HV/MV substations. This system may implement according to the state of the art a GSM mobile connection, or power line communications. In the case of power line communications, at least two capacitive or inductive couplers will have to be installed (with a typical cost of $20000 €$ ), whereas the GSM communication system has a typical cost of $1800 €$ per transformer. The latter will be therefore used in the following calculations in order to improve feasibility.

Finally, the implementation of smart grid techniques on the power distribution network may require the addition of an embedded computer (with a typical cost of $500 €$ ), a UPS (with a typical cost of $500 €$ ), and two motor driven MV load switches, which may be remotely used in order to perform switching operations (typical cost: $7000 €$, switchgear not included) [1].

The resulting cost for the deployment of the aforementioned equipment at a single MV/LV transformer reaches $17100 €$. For the single feeder of Fig. 2, this corresponds to a total of $307800 €$, which is prohibitive considering the large number of feeders used in even a medium scale city.

\section{Selective Automation Upgrade for Reliability IMPROVEMENT AND LOSS REDUCTION PURPOSES}

\section{A. Reliability Improvement}

Reliability improvement depends greatly on the time needed for fault isolation and power restoration in the case of an outage. The basic challenge for the DSO is to ensure the immediate fault detection within the shortest possible segment of the feeder that undergoes the outage. Usually, the shortest possible segment for fault detection is the line segment between two adjacent distribution transformers. Therefore, fault isolation requires the opening of the respective MV load switches at the corresponding ends of the segment. As already mentioned, these switches are in most cases manual in the DNs. Thereby, fault isolation is implemented by the DSO via sequential movements of technical personnel across the feeder, along with corresponding manual switching operations. In Fig. 3 the possible manual interferences for fault isolation are presented for the simple case of a radial feeder. The basic disadvantage of DNs that lack automation and decentralized self-management constitutes in crucial delays when immediate management of the network's components is required. PPC reports an average time of $2 \mathrm{hrs}$ for the manual fault isolation in large urban DNs in Greece, due to traffic delays and manual switching operations.

For these reasons, automation upgrade with respect to reliability improvement, in feeders with only manual load switches, should initially be focused on upgrading the functionality of these switches in order to permit remote control. The benefits in this case correspond to the reduction of the time needed for fault isolation and the respective reliability improvement. Naturally, the level of the automation upgrade will also affect the magnitude of these benefits. For example, it is expected that the installation of remote controlled load switches in every step-down transformer could ensure power restoration in the minimum possible time. The notion of "Grid Smartness" is determined in this case by the algorithm that will be selected to control the sequence of the appropriate switching operations, while the automation upgrade constitutes a prerequisite towards the implementation of a smart grid.

For the purposes of this work, it is considered that the multiagent system (MAS) presented in [2] is available for the process of the automated fault isolation and the respective power restoration after an outage in a DN. Using this MAS as a basis, different investments on the DN are investigated, according to the selective upgrade of specific DN nodes, and the respective feasibility is studied for each different test case.

\section{B. Loss Reduction}

Loss reduction in DNs is implemented by network reconfigurations. The idea of utilizing the structure of a DN in order to cause loss reduction under normal operating conditions was first formulated by Merlin and Back [3] in 1975. The problem has been investigated by many researchers since then, and a great number of algorithms have been proposed during the last decades. Nevertheless, most of these aforementioned algorithms deal with the problem only for fixed operating conditions, since they propose a reconfigured topology derived for constant loading conditions. On the other hand, the load curves of real DNs indicate continuous load variations; hence a question arises regarding the optimum reconfigured topology given these variations. Some researchers [4]-[10] argue that the DN reconfiguration for loss minimization should adapt to load variations; however, the subject of the minimum tolerance of load variations that would justify a new reconfiguration has 


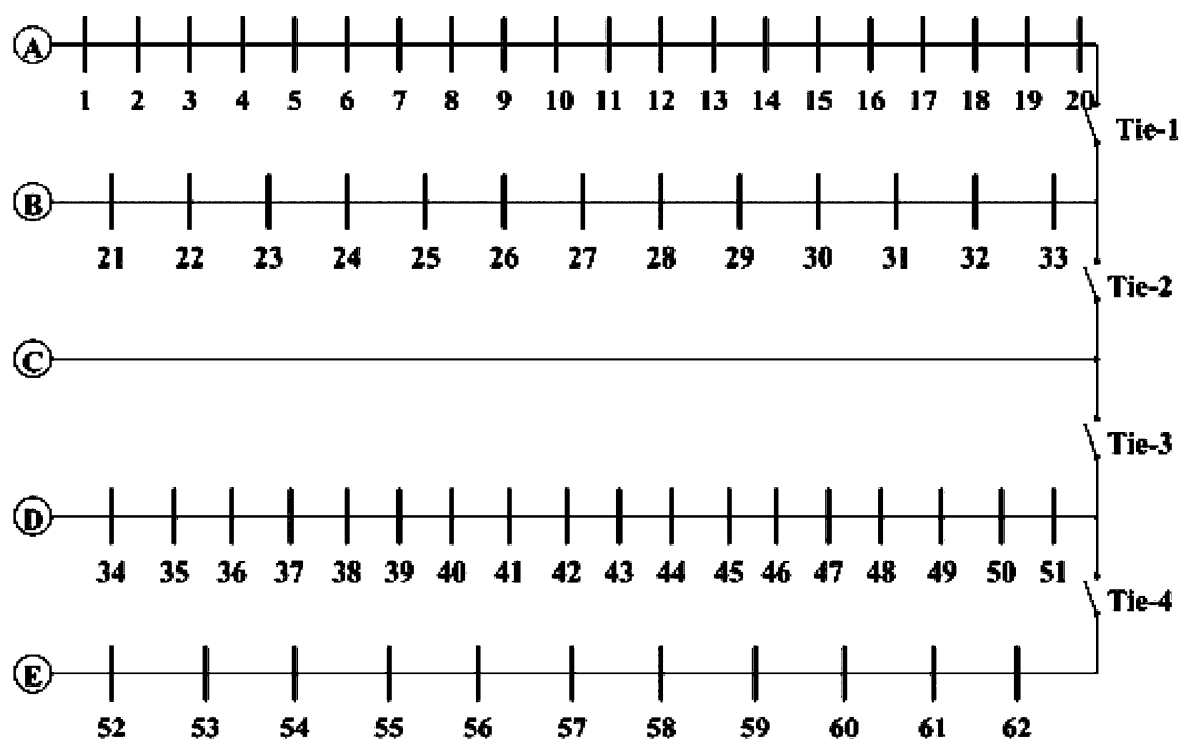

Fig. 4. Test case DN segment.

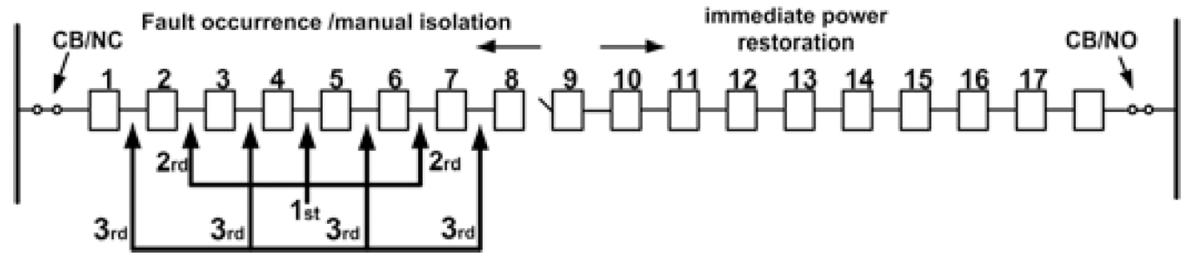

Fig. 5. Sequential switching operations for fault isolation in the presence of remotely controlled load switches in the middle of the feeder.

not been fully investigated. It is of major importance to define explicit criteria regarding the frequency of network reconfiguration due to load variations. Limited reconfigurations may result in a significant divergence from the optimum solution concerning loss reduction. On the other hand, seeking for an optimum reconfiguration after any load change would cause large computation effort and numerous switching operations. The former is considered ineffective for real-time applications, while the latter is expected to shorten the life cycle of the load switches.

Loss minimization by network reconfiguration involves an exhaustive search of the suitable pairs of switching operations. Based on the problem formulation [11], for a network with $n$ load switches the maximum number of switching operations that should be examined for the static problem are $2^{n}$. Naturally, the complexity of the problem increases excessively for real operating conditions, taking into account the network load variations. In [12] the authors present an algorithm that incorporates load alterations in the reconfiguration process for loss reduction. The results of this algorithm will serve as a basis for the respective feasibility studies concerning the test cases of this work.

\section{Test Cases Regarding Selective AUTOMATION UPGRADE}

\section{A. Reliability Improvement by Selective Automation Upgrade}

In order to justify that selective automation could improve the management of DNs, the network in Fig. 4 is utilized as
TABLE I

INPUT DATA FOR RELIABILITY ANALYSIS

\begin{tabular}{c|c}
\hline Number of step-down transformers & 62 \\
\hline Network nominal power (KVA) & 42,44 \\
\hline Total network length (km) & 20.743 \\
\hline Failure rate (f/yr*km) & 0.1 \\
\hline Repair time (hrs) & 2 \\
\hline
\end{tabular}

the test case in the following analysis. The network consists of four underground feeders (feeders A, B, D, and E) along with a standby feeder (C) that is used to serve some, or all loads of the feeder that has suffered an outage. Simulations regarding reliability analysis were implemented in Neplan software [13]. For the initial state regarding the network automation level it is assumed that only manual sectionalizing switches exist.

Based on the typical manual procedure followed by the DSO personnel for the fault isolation at a feeder, as illustrated in the single feeder of Fig. 5, it is evident that the installation of remote switches at both sides of the middle substation of the feeder could cause immediate power restoration to almost half consumers. In Fig. 5 it is assumed that the fault occurs at the 1st half of the feeder; therefore remote controlled switches at feeder \#9 are operated properly to restore power to the healthy half of the DN segment. For the other half of the DN segment, manual operations are still needed until the fault is isolated between adjacent substations. In the test case studied here, it is assumed that two remote switches are installed in the middle substation of each feeder in Fig. 4. In Table I the reliability data imported 
TABLE II

Reliability ANALysis Results For Selective Automation UpgRade

\begin{tabular}{|c|c|c|c|c|c|c|c|c|c|}
\hline & \multirow{2}{*}{ Reliability indices } & \multicolumn{4}{|c|}{ manual switches } & \multicolumn{4}{|c|}{$\begin{array}{l}\text { two remote switches to each feeder - } \\
\text { installation at the middle substation }\end{array}$} \\
\hline & & feeder A & feeder B & feeder D & feeder E & feeder A & feeder B & feeder D & feeder E \\
\hline SAIFI & System average interruption frequency index (1/yr) & 0.623 & 0.491 & 0.534 & 0.426 & 0.623 & 0.491 & 0.534 & 0.426 \\
\hline SAIDI & System average interruption duration index (min/yr) & 74.767 & 58.947 & 64.128 & 51.073 & 33.61 & 27.36 & 30.196 & 23.06 \\
\hline CAIDI & Customer average interruption duration index (h) & 2 & 2 & 2 & 2 & 0.899 & 0.928 & 0.942 & 0.903 \\
\hline $\mathrm{F}$ & System load interruption frequency $(1 / y r)$ & 0.623 & 0.491 & 0.534 & 0.426 & 0.623 & 0.491 & 0.534 & 0.426 \\
\hline $\mathrm{T}$ & System load interruption mean duration (h) & 2 & 2 & 2 & 2 & 1.827 & 1.903 & 1.904 & 1.887 \\
\hline $\mathrm{W}$ & Total expected energy not supplied (MWh/yr) & 4.293 & 1.854 & 3.596 & 1.549 & 1.952 & 0.882 & 1.687 & 0.668 \\
\hline
\end{tabular}

in the software for the simulations are shown, and in Table II the results concerning reliability improvement by this selective approach are presented.

The reliability input data in Neplan for first order failure modes, i.e., single failures, are mainly the failure rate and repair time. The respective output data consist of the failure rate and the duration of each power outage. Additional output data correspond to the values for the system reliability indices and the expected energy not supplied (EENS). Owing to their low probability of occurrence, multiple outages are neglected.

The results presented in Table II indicate that the automation upgrade of the sectionalizing switches at the middle substation of each feeder only, could decrease system average interruption duration index (SAIDI) and EENS index even by 50\%. Furthermore, the annual benefit regarding the EENS index for the entire network segment can reach $6103 \mathrm{kWh}$, which constitute in financial terms an annual benefit of approximately $20000 €$. For the estimation of this benefit, the saved $\mathrm{kWhs}$ have been rated with the value of the value of lost load (VLL) index [2]. The investment cost for this selective automation upgrade could be paid back within a time period of two years, considering the cost for the substitution of eight manual sectionalizing switches with remote controlled ones.

\section{B. Loss Reduction by Selective Automation Upgrade}

The investigation regarding the influence of load variations on the optimum reconfiguration for the test case DN segment of Fig. 4 has been implemented by applying the algorithm presented in [12]. For the definition of the initial static problem for a fixed operational point it was assumed that the loads at every node take the mean load values of month of March. The algorithm was implemented for 10000 scenarios with different load composition. Load variation was simulated by stochastic uniform distribution and the utilized value for the deviation was set to $\pm 50 \%$.

The results indicate that the sectionalizing switches that define the optimum reconfiguration for the initial mean load values participate to the majority of different static problems with altered loads. In Table III the participating percentages of all possible switches that could be involved in optimum reconfiguration, regardless of the load composition, are shown. Branches marked bold correspond to the ones of the initial static problem. It is obvious by the results shown in Table III that the optimum
TABLE III

LOAD VARIATION AND CORRESPONDING SWITCHING OPERATIONS

\begin{tabular}{|c|c|}
\hline Tie switch & $\begin{array}{c}\text { Percentage of branch } \\
\text { participation in minimum loss } \\
\text { configurations }\end{array}$ \\
\hline \multirow{5}{*}{ Tie-1 } & $13-14$ with $0.25 \%$ \\
\hline & $14-15$ with $14.17 \%$ \\
\hline & 15-16 with $57.96 \%$ \\
\hline & $16-17$ with $27.52 \%$ \\
\hline & $17-18$ with $0.1 \%$ \\
\hline \multirow{4}{*}{ Tie-2 } & 26-27 with $7.57 \%$ \\
\hline & $27-28$ with $87.11 \%$ \\
\hline & $28-295.31 \%$ \\
\hline & $29-30$ with $0.01 \%$ \\
\hline \multirow{4}{*}{ Tie-3 } & 43-44 with $6.74 \%$ \\
\hline & 44-45 with $69.28 \%$ \\
\hline & 45-46 with $23.84 \%$ \\
\hline & $46-47$ with $0.14 \%$ \\
\hline \multirow{4}{*}{ Tie-4 } & $59-60$ with $0.11 \%$ \\
\hline & $60-61$ with $73.95 \%$ \\
\hline & 61-62 with 15\% \\
\hline & $52-63$ with $0.05 \%$ \\
\hline
\end{tabular}

reconfiguration derived for a fixed operational point is expected to keep defining the optimum solution for the most of the examined scenarios, even if the magnitude of loads varies within a range of $\pm 50 \%$ from the initial mean values.

On the other hand, in some scenarios, different branches from the aforementioned ones of the initial static problem participate in the optimum reconfigurations. The aggregated group of the branches in Table III determines the optimum real-time management of the network for loss minimization. It is reasonable to assume that the automation upgrade of the respective nodes in Table III should ensure remote network reconfiguration under any load composition that is defined by real load curves. Such an investment approach results in a significant lower cost when compared to the automation upgrade of all load switches of the network segment under study. 


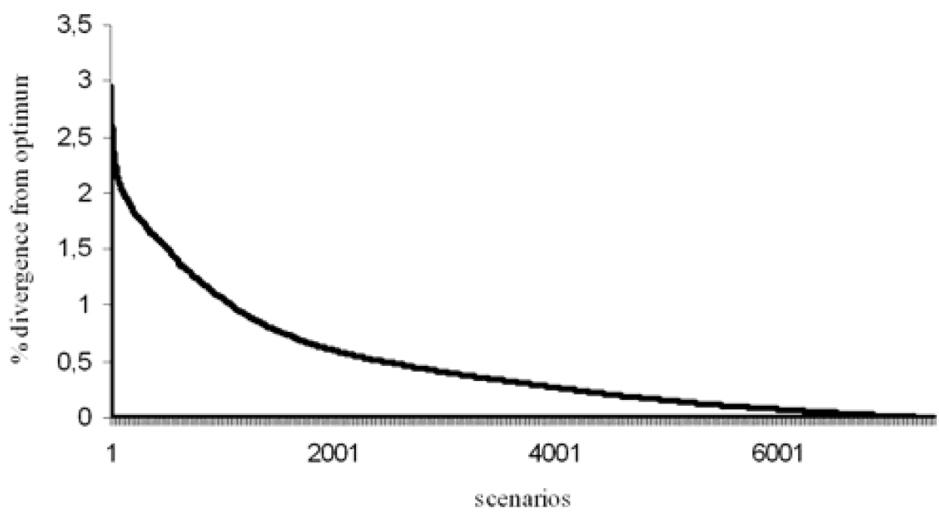

Fig. 6. Differences in loss reduction between the initial solution and a scenario with different optimum solution.

TABLE IV

REliability ANALYsis Results for AdDITIONAL Selective Automation UpgRade

\begin{tabular}{|c|c|c|c|c|c|c|c|c|c|c|c|c|}
\hline \multirow[t]{2}{*}{ Reliability indices } & \multicolumn{4}{|c|}{ manual switches } & \multicolumn{4}{|c|}{$\begin{array}{l}\text { one remote switch to each feeder - } \\
\text { installation at the middle substation }\end{array}$} & \multicolumn{4}{|c|}{$\begin{array}{c}\text { additional automation upgrade of the } \\
\text { branch with the highest participating } \\
\text { percentage }\end{array}$} \\
\hline & feeder $\mathrm{A}$ & feeder B & feeder D & feeder E & feeder A & feeder B & feeder D & feeder E & feeder A & feeder B & feeder D & feeder E \\
\hline SAIFI (1/yr) & 0.623 & 0.491 & 0.534 & 0.426 & 0.623 & 0.491 & 0.534 & 0.426 & 0.623 & 0.491 & 0.534 & 0.426 \\
\hline SAIDI (min/yr) & 74.767 & 58.947 & 64.128 & 51.073 & 33.61 & 27.36 & 30.196 & 23.06 & 23.91 & 24 & 23.44 & 19.39 \\
\hline CAIDI (h) & 2 & 2 & 2 & 2 & 0.899 & 0.928 & 0.942 & 0.903 & 0.64 & 0.815 & 0.731 & 0.759 \\
\hline $\mathrm{F}(1 / \mathrm{yr})$ & 0.623 & 0.491 & 0.534 & 0.426 & 0.623 & 0.491 & 0.534 & 0.426 & 0.623 & 0.491 & 0.534 & 0.426 \\
\hline $\mathrm{T}(\mathrm{h})$ & 2 & 2 & 2 & 2 & 1.827 & 1.903 & 1.904 & 1.887 & 1.649 & 1.765 & 1.799 & 1.799 \\
\hline W (MWh/yr) & 4.293 & 1.854 & 3.596 & 1.549 & 1.952 & 0.882 & 1.687 & 0.668 & 1.429 & 0.769 & 1.272 & 0.552 \\
\hline
\end{tabular}

However, in a selective automation upgrade, each selection of a node to be upgraded has to be fully justified in terms of feasibility. Therefore, an ad hoc investigation has been performed, in order to confirm that the upgrade of all other nodes except for the ones that present the highest participating percentages in Table III is not essential. For this purpose, it was again assumed that only the nodes with the highest participating percentages are upgraded (the ones marked bold in Table III), and the divergence from the optimum reconfiguration solution was calculated for all the scenarios in terms of loss reduction.

In Fig. 6 the scenarios for which the selected upgraded nodes did not constitute the optimum reconfiguration are presented, along with the divergence with respect to the actual optimum solution for every case. As shown in Fig. 6, the greatest divergence of the selected solution as compared to the optimum one for all scenarios considered is only $2.5 \%$. Furthermore, in almost 6000 of total 7183 scenarios for which a difference exists in loss reduction as compared to the optimum solution, this difference is lower than $1 \%$, and thus it may be considered negligible. The crucial conclusion derived by these results is that the reconfigured topology defined by the branches with the highest participating percentages constitutes the optimum or near optimum solution under normal load variations, and hence normal operating conditions. In this case, the annual benefit regarding the EENS index for the entire network segment could result in $4677 \mathrm{kWh}$. In financial terms, this benefit corresponds to approximately $19000 €$.

It is proposed to consider the results in Table III as a guideline that prioritizes automation upgrade when real-time loss re- duction is desired. Selective automation upgrade in such a case should refer to sequential automation upgrade starting from the branch with the highest participating percentage at each feeder towards the one with the lowest one.

\section{Combined Selective Automation Upgrade}

In the previous subsections, the selective upgrade of the network segment under study is investigated for two distinct cases of smart grid functionality. For each case, the results indicate different nodes to be upgraded. The next step is to combine the two solutions, assuming that the selective upgrade concerns all the nodes proposed by the previous approaches.

Table IV illustrates how reliability could be further improved due to this additional automation upgrade. It is observed by the results of Table IV that when upgrading two more sectionalizing switches, at both ends of the respective branch, the SAIDI index could be improved even up to approximately $70 \%$ for some feeders in comparison to the 50\% of the first approach with the automation upgrade concerning only the middle substations of all feeders involved.

A brief feasibility study indicates that the combined solution upgrades could result in an annual benefit regarding EENS index up to $7290 \mathrm{kWh}$ instead of $6103 \mathrm{kWh}$ that resulted by the first selective upgrade. This additional improvement equals to an annual benefit by reliability improvement of approximately $30000 €$

The annual benefit by loss reduction as estimated in [1] could be up to $2400 €$ when the network is reconfigured once a day for a specific time period. The total annual benefit by both reli- 


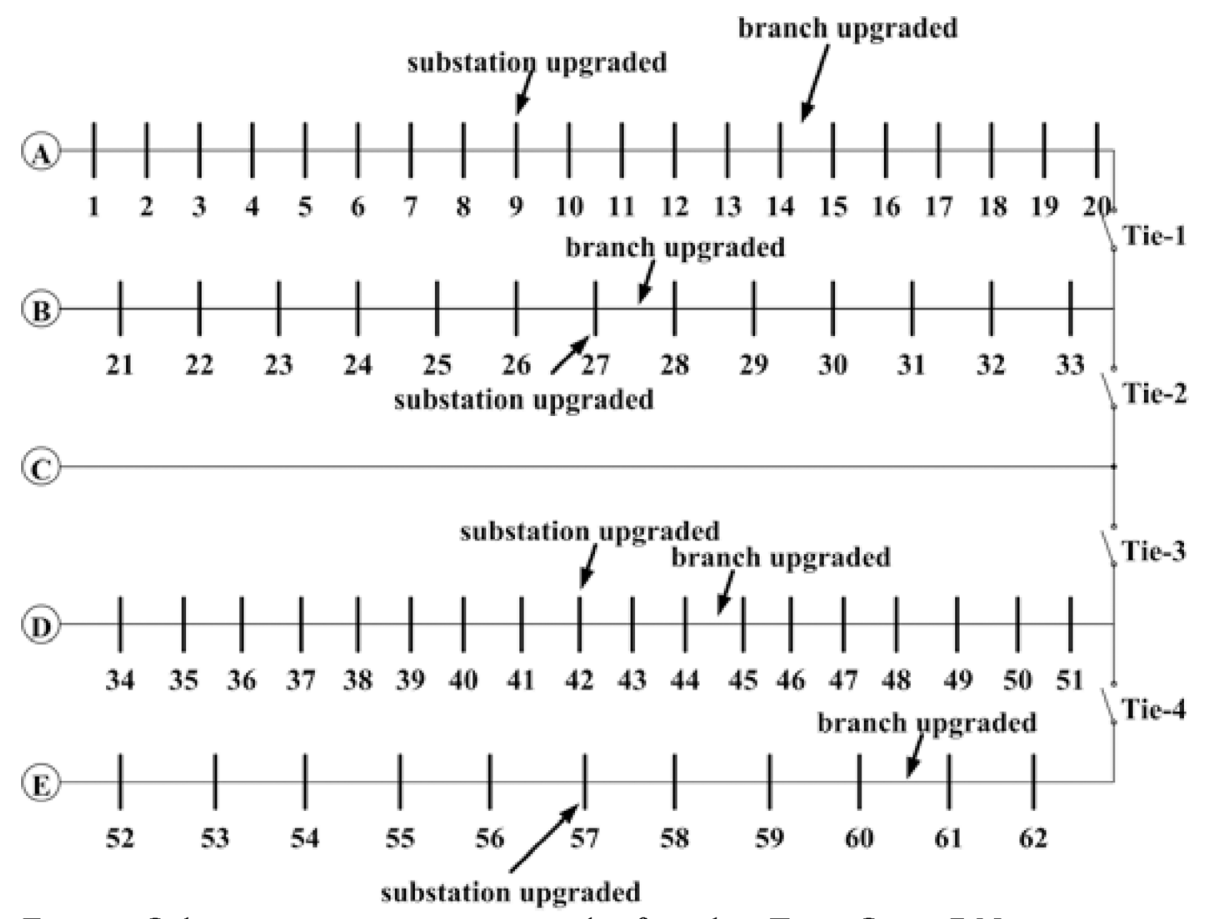

Fig. 7. Selective automation upgrade for the test case DN segment-automation upgrade for both reliability improvement and loss reduction.

ability improvement and loss reduction is estimated to $32400 €$. In Fig. 7 the upgraded network is illustrated concerning both criterions for reliability improvement and real-time near optimum management for loss reduction. The total cost for the proposed selective automation upgrade is estimated to approximately $70000 €$ (56000€ regarding the installation of four remote controlled switches in every feeder and $14400 €$ regarding the installation of the GSM communication system at the upgraded nodes of the network). Based on the annual benefit by reliability improvement and loss reduction resulted by the upgrade presented in Fig. 7 and analyzed previously, the payback time is estimated to less than 2.2 years.

\section{CONCLUSION}

The cost of the essential upgrades could prove to be a big barrier for the large scale implementation of smart grid techniques in power distribution networks. This work provides guidelines towards the selective automation upgrade in such networks, so as to determine the prerequisites for specific targeted actions, ensuring at the same time the feasibility of the respective investments. Upgrades have been performed in DNs with no previous automation capabilities, in order to achieve real-time management for reliability improvement and loss reduction. The scenarios studied involved these two targets both individually as well as combined. Results show that in every case a satisfactory equilibrium may be achieved between investment cost and functionality.

\section{ACKNOWLEDGMENT}

The authors would like to thank Messrs. S. Fahouridis and A. Zafirakis of the PPC for providing the needed data that enabled this study to be conducted successfully.

\section{REFERENCES}

[1] A. S. Bouhouras, G. T. Andreou, and D. P. Labridis, "Feasibility study of the implementation of A.I. automation techniques in modern power distribution networks," Electr. Power Syst. Res., vol. 8, no. 5, pp. 495-505, May 2010.

[2] A. S. Bouhouras, D. P. Labridis, and A. G. Bakirtzis, "Cost/worth assessment of reliability improvement in distribution networks by means of artificial intelligence," Int. J. Electr. Power Energy Syst., vol. 32, no. 5, pp. 530-538, Jun. 2010.

[3] A. Merlin and H. Back, "Search for a minimal-loss operating spanning tree configuration in an urban power distribution system," in Proc. 5th Power Syst. Comput. Conf. (PSVV), Cambringe, U.K., 1975, pp. 1-18.

[4] R. P. Broadwater, A. H. Khan, and H. E. Shaalan, "Time varying load analysis to reduce distribution losses through reconfiguration," IEEE Trans. Power Del., vol. 8, pp. 294-300, 1993.

[5] C. S. Chen and M. Y. Cho, "Energy loss reduction by critical switches," IEEE Trans. Power Del., vol. 8, no. 3, pp. 1246-1253, 1993.

[6] G. J. Peponis, M. P. Papadopoulos, and N. D. Hatziargyriou, "Distribution network reconfiguration to minimize resistive line loss," IEEE Trans. Power Del., vol. 10, no. 3, pp. 1338-1342, 1995.

[7] P. A. Vargas, C. L. Filho, and F. J. Von Zuben, "On-line approach for loss reduction in electric power distribution networks using learning classifier systems," in Advances in Learning Classifier Systems. Berlin, Germany: Springer, 2002, vol. 2321/2002, pp. 271-307.

[8] K. Y. Huang and H. C. Chin, "Distribution feeder energy conservation by using heuristics fuzzy approach," Int. J. Electr. Power Energy Syst., vol. 24, no. 6, pp. 439-445, 2002.

[9] E. L. Lopez, H. O. Opazo, L. Garcia, and P. Bastard, "Online reconfiguration considering variability demand: Applications to real networks," IEEE Trans. Power Syst., vol. 19, no. 1, pp. 549-543, 2002.

[10] E. A. Bueno, C. Lyra, and C. Cavelluci, "Distribution network reconfiguration for loss reduction with variable demands," in Proc. 2004 IEEE/PES Transm. Distrib. Conf. Expo. Latin America, São Paulo, Brazil, pp. 384-389.

[11] H. P. Schmidt, N. Ida, N. Kagan, and J. C. Guaraldo, "Fast reconfiguration of distribution systems considering loss minimization," IEEE Trans. Power Syst., vol. 20, no. 3, pp. 1311-1319, 2005.

[12] A. S. Bouhouras, G. T. Andreou, and D. P. Labridis, "Reliability improvement in distribution networks by the upgrade of critical switches," in IEEE Proc. 7th Int. Conf. Eur. Electr. Market (EEM 2010), Madrid, Spain, June 23-25, 2010, p. 6.

[13] Neplan Version 5.1.7 1988-2003, BCP Busarello+Cott+Partner Inc. 
Aggelos S. Bouhouras was born in Thessaloniki, Greece, on May 24, 1980. He received the Dipl.-Eng. and Ph.D. degrees from the Department of Electrical and Computer Engineering, Aristotle University of Thessaloniki, in 2005 and 2010, respectively.

Currently he is a Research Associate in the Department of Electrical and Computer Engineering, Aristotle University of Thessaloniki. His research activities concern power distribution system analysis with emphasis on loss reduction and reliability improvement, and smart grids.

Georgios T. Andreou (S'98-A'02-M'08) was born in Thessaloniki, Greece, on August 16, 1976. He received the Dipl.-Eng. and Ph.D. degrees from the Department of Electrical and Computer Engineering, Aristotle University of Thessaloniki, in 2000 and 2006, respectively.

Currently he is a Lecturer in the Department of Electrical and Computer Engineering, Aristotle University of Thessaloniki. His special interests are power system analysis with special emphasis on the simulation of transmission and distribution systems, electromagnetic and thermal field analysis, and power line communications.
Dimitris P. Labridis (S'88-M'90-SM'00) was born in Thessaloniki, Greece, on July 26, 1958. He received the Dipl.-Eng. and the Ph.D. degrees from the Department of Electrical and Computer Engineering, Aristotle University of Thessaloniki, Greece, in 1981 and 1989 respectively.

Since 1986 he has been with the Electrical Engineering Department, Aristotle University of Thessaloniki, where he is currently a Professor. His research interests are power system analysis with special emphasis on the simulation of transmission and distribution systems.

Anastasios G. Bakirtzis (S'77-M'79-SM'95) received the Dipl. Eng. Degree from the Department of Electrical Engineering, National Technical University, Athens, Greece, in 1979 and the M.S.E.E. and Ph.D. degrees from Georgia Institute of Technology, Atlanta, in 1981 and 1984, respectively.

Since 1986 he has been with the Electrical Engineering Department, Aristotle University of Thessaloniki, Greece, where he is currently a Professor. His research interests are in power system operation, planning, and economics. 